\section{Sound synthesis by minicomputer: System PHON}

\author{
MICHAEL G. STROBEL \\ University of Montreal, Montreal, Canada
}

and

\author{
CHING Y. SUEN \\ Department of Computer Science \\ Concordia University, Montreal, Canada
}

The present paper describes a flexible technique to produce and reliably reproduce sound patterns of arbitrary complexity under complete control of the operator. Waveforms can be copied from live source or generated by hand. Intensity, frequency, duration, and number of components, as well as cycling patterns can be specified. The digitized data of the signal can be easily stored or transported to other equipment in analog or digital forms. In all stages, there is visual control and temporal "freezing" of the signal wave is possible. The limitations of core memory are largely overcome by programming techniques.

The main advantage of using computer techniques in the generation stage is the compatibility of the phonetic patterns with electronic synthesizers, thus bypassing the technical difficulties of recording and reproducing arbitrary waveforms of high complexity. In fact, the program called "PHON," to be described here, contains a software synthesizer which can readily be used in ROM chips in combination with a microprocessor.

PHON is a PDP-12 (8K) computer program capable of extracting the characteristics of a continuous waveform as digitized data points from which complex sound patterns can be synthesized by a computer.

As a simple but typical example, suppose the sound of the letter " $A$ " is to be produced by a machine. Connecting a microphone to one of the A/D input channels on a PDP-12. and pronouncing the letter " $A$ " will produce the waveform representation of the spoken sound on the screen. Inspection shows that the sound " $\mathrm{A}$ " consists basically of two patterns, one appearing at the beginning and the other toward the end of the spoken letter. These two patterns correspond to the two phonemes which make up the letter sound " $\mathrm{A}$ " in the English language: /e/ and / $/ /$. The waveform of the phonemes appears as cyclic repetitions of a characteristic whose form, frequency, and amplitude can be seen clearly on the screen when the wave is frozen. By cutting the required segment from the continuous wave and storing the digitized sample, one has the minimal amount of information necessary to reproduce the waveform by simply repeating the characteristic as long as desired or necessary.

The human ear needs a limited number of cycles to activate the physiological mechanism for sound identification or recognition. The minimum number of cycles can be found quickly by continuous adjustment while listening to the repeated utterance of the wave characteristics through an earphone, connected to the slave-scope contact and to an audio-amplifier.

When satisfied with the performance, the sample, together with the empirically determined number of cycles (repetitions), can be stored permanently on LINC-tape. The operation of selecting a "good" characteristic may involve several attempts at segmenting, smoothing, and reshaping, all of which are facilitated by the appropriate controls from the computer panel.

Having identified, extracted, and stored the phoneme data, the process of speech synthesis can be attempted by joining the appropriate phonemes together. The output can be monitored, using switch controls, by listening through the earphones or a loudspeaker. During this stage, it may again be necessary to modify the characteristics for better quality of onset and termination. When satisfied with the data, the operator can store the sound patterns in memory for further processing.

Since LINC-tape is not a universal storage device and frequently other machines (e.g., a microprocessor with readonly memories) are the final destination of the digitized samples, a subroutine is included in the PHON program so that the required data can be punched on paper tape. Furthermore, the format of the data can be packed (i.e., two points to a word) into a four-digit octal number in order to save space. Where there is a need for more precise data of a waveform appearing on the scope, the data can be punched directly as viewed and selected as three-digit signed decimal numbers.

The program requires $2000_{8}$ locations; $2000_{8}$ locations are reserved for the assembly of patterns and sound synthesis; $14000_{8}\left(6144_{10}\right)$ locations are available for analog sampling; the last $400_{8}$ locations, however, will be overwritten in the case of a tape-to-memory operation. Sampling is controlled at 80 microsec from the $\mathrm{KW}-12$ clock. The sampling rate can easily be modified within the program.

The PHON program is complemented by a PACK program which allows the characteristics stored on individual tape blocks to be compacted into continuous data blocks for indexed filing to the DIAL-MS system so that the data can be added to a synthesizer program in binary.

This synthesizer consists of a table of the addresses of various characteristics and an input routine so that these characteristics can be activated by commands from an input device. When the letter " $A$ " is pressed on the Teletype, the synthesizer receives the ASCII code (301) for the letter "A." Upon receipt of this code, the synthesizer first looks up the location and repetitions of the two constituent phonemes $/ \mathrm{e} /$ and $/ \mathrm{l} /$ and then generates them in sequence.

The synthesis process involves nothing more than separating the two data points packed in one word, shifting for magnification and display on the screen, or feeding them to a D/A output device. The information necessary to build the tables of addresses, number of repetitions, as well as the duration of the sounds, is provided by the PHON program and the PACK program.

The program described here has been successfully employed in a talking typewriter of the SPELLEX system for the blind (Suen \& Beddoes, 1974, 1975). Visual feedback available for the sighted from the typewriter output is transformed into sounds of the spoken letter for the blind. The keyboard of an ordinary typewriter is interfaced to an ASCII encoder from which the microprocessor composes the letter according to a phoneme table. Data are then retrieved from ROM and cycled sufficiently long to permit auditory identification, yet fast enough to permit paced typing. The PHON program made it possible to produce data for any desired language such that the SPELLEX system can now be produced in polyglott versions.

Availability. A manual of operations for System PHON is available at a cost of $\$ 4.00$ to cover printing and handling expenses. Program listing is available also. Address requests to Professor Chin Y. Suen, Room H963, Department of Computer Science, Concordia University, 1455 de Maisonneuve Blvd. West, Montreal, Quebec, Canada H3G IMB.

\section{REFERENCES}

Suen, C. Y., \& Beddoes, M. P. Development of SPELLEXa talking typewriter for the blind. Proceedings of the 27th Annual Conference on Engineering, Medicine, and Biology, 1974, 16, 348.

Suen, C. Y., \& Beddoes, M. P. Spelled speech as an audio output for the LEXIPHONE reading machine and the SPELLEX talking typewriter for the blind. Research Bulletin of the American Foundation for the Blind, 1975, 29, 51-66. 